\title{
New Pioglitazone Metabolites and Absence of Opened-Ring Metabolites in New $\mathbf{N}$-Substituted Thiazolidinedione ${ }^{\text {午 }}$
}

\author{
Michel Leandro Campos, Letícia Bonancio Cerqueira, Bruna Cristina Ulian Silva, \\ Taísa Busaranho Franchin, Marina Rocha Galdino-Pitta, Ivan Rocha Pitta, \\ Rosângela Gonçalves Peccinini, and Roberto Pontarolo
}

\begin{abstract}
Department of Pharmacy, Universidade Federal do Paraná, Curitiba, Paraná, Brazil (M.L.C., L.B.C., R.P.); Department of Natural Active Principles and Toxicology, Faculdade de Ciências Farmacêuticas, São Paulo University (UNESP), Araraquara, São Paulo, Brazil (B.C.U.S., T.B.F., R.G.P.); and Laboratory of Design and Drug Synthesis, Universidade Federal de Pernambuco, Pernambuco, Brazil (M.R.G.-P., I.R.P.)
\end{abstract}

Received November 5, 2017; accepted March 30, 2018

\section{ABSTRACT}

Thiazolidinediones (TZDs) are drugs used to treat type 2 diabetes mellitus; however, several safety concerns remain regarding the available drugs in this class. Therefore, the search for new TZD candidates is ongoing; metabolism studies play a crucial step in the development of new candidates. Pioglitazone, one of the most commonly used TZDs, and GQ-11, a new $\mathbf{N}$-substituted TZD, were investigated in terms of their metabolic activity in rat and human liver microsomes to assess their metabolic stability and investigate their metabolites. Methods for preparation of samples were based on liquid-liquid extraction and protein precipitation. Quantitation was performed using liquid chromatography (LC)-tandem mass spectrometry, and the metabolite investigation was performed using ultraperformance LC coupled to a hybrid quadrupole-time of flight mass spectrometer. The predicted intrinsic clearance of GQ-11 was 70.3 and $46.1 \mathrm{ml} / \mathrm{kg}$ per minute for rats and humans, respectively. The predicted intrinsic clearance of pioglitazone was 24.1 and $15.9 \mathrm{ml} / \mathrm{kg}$ per minute for rats and humans, respectively. The pioglitazone metabolite investigation revealed two unpublished metabolites (M-D and M-A). M-A is a hydration product and may be related to the mechanism of ring opening and the toxicity of pioglitazone. The metabolites of GQ-11 are products of oxidation; no ring-opening metabolite was observed for GQ-11. In conclusion, under the same experimental conditions, a ring-opening metabolite was observed only for pioglitazone. The resistance of GQ-11 to the ring opening is probably related to $N$-substitution in the TZD ring.

\section{Introduction}

Thiazolidinediones (TZDs) are drugs used to treat type 2 diabetes mellitus. They are also called glitazones, and the most commonly used of these compounds include troglitazone, rosiglitazone, and pioglitazone (Saltiel and Olefsky, 1996; Naim et al., 2017). TZDs are a class of oral insulin-sensitizing drugs whose mechanism of action involves the activation of peroxisome proliferator-activated receptor $\gamma$ (Hauner, 2002; Stumvoll, 2003). Troglitazone was the first launched thiazolidinedione, but it was withdrawn from the market for severe and sometimes fatal hepatotoxicity (Scheen, 2003). Rosiglitazone is available in the United States, whereas in Europe, its approval was withdrawn owing to a significant increase in myocardial infarction among users (Alemán-González-Duhart et al., 2016); hepatotoxicity was also reported (Al-Salman et al., 2000; Forman et al., 2000). Pioglitazone (Fig. 1A) use in the United States is

This work was supported by the State of Parana Research Foundation (Fundação Auracária), National Council for Scientific and Technological Development (CNPq), São Paulo Research Foundation (FAPESP process 2016/04927-0), and Coordination for the Improvement of Higher Education Personnel.

https://doi.org/10.1124/dmd.117.079012.

$\$$ This article has supplemental material available at dmd.aspetjournals.org. restricted as it may cause urinary bladder cancer (Alemán-González-Duhart et al., 2016). In addition, hepatotoxicity cases have occurred (Maeda, 2001; Marcy et al., 2004), including one fulminant hepatic failure (Chase and Yarze, 2002). Given these adverse reactions, the search for safer TZDs is ongoing. Several new candidates have been reported in the literature (Imran et al., 2007; Naim et al., 2017). Most of these candidates possess substitutions in the carbon 5 of the thiazolidinedione ring only; however, substitutions in the nitrogen at position 3 have shown good pharmacologic activities in models of diabetes (Mourao et al., 2005) and atherosclerosis (Cesar et al., 2015). Mourão et al. (2005) published a study on the synthesis of 5-benzylidene and 5-acridinylidene derivatives, both of which were $N$-3 substituted; these compounds showed hypoglycemic activity in mice. Many other researchers have used similar approaches with promising outcomes (da Costa Leite et al., 2007; Barros et al., 2010; Araújo et al., 2011; Amato et al., 2012; Santin et al., 2013a,b; Cesar et al., 2015; Rudnicki et al., 2016; Silva et al., 2016).

Metabolism studies are an important step in the development of new compounds (Kostiainen et al., 2003). In addition, because of toxicity concerns, investigation of possible metabolites is preferably performed soon in the development process, avoiding delays in the later phases (Brandon et al., 2003; Masimirembwa et al., 2003; Beuck et al., 2011). This type of research and information is crucial since active metabolites

ABBREVIATIONS: $\mathrm{CL}_{h}$, hepatic clearance; $\mathrm{CL}_{\text {int }}$, intrinsic clearance; $\mathrm{ESI}$, electrospray ionization; GQ-11, 5-(indol-3-ylmethylene)-3-(4-methylbenzyl)thiazolidine-2,4-dione; HLM, human liver microsome; LC, liquid chromatography; MS, mass spectrometry; RLM, rat liver microsome; TZD, thiazolidinedione. 
<smiles>CCc1ccc(CCOc2ccc(CC3SC(=O)NC3=O)cc2)nc1</smiles>

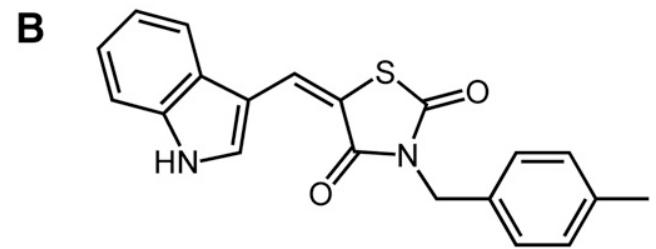

Fig. 1. Structures of pioglitazone (A) and the new thiazolidinedione GQ-11 (B).

are one of the main causes of unexpected safety issues in the research and development of new drug candidates (Baillie et al., 2002; Leclercq et al., 2009). With the recent publication of the Guidance for Metabolite Safety Testing (FDA, 2008), metabolite investigations have become even more important (Baillie, 2008; Leclercq et al., 2009). Considering TZDs, it was proposed that the ring opening may be related to the formation of reactive metabolites of troglitazone (Kassahun et al., 2001). This ring-opening mechanism is common to other TZDs (Alvarez-Sánchez et al., 2006). Shen et al. (2003) first reported the ring opening of pioglitazone but only in dog microsomes; Baughman et al. (2005) described these metabolites in rat and human microsomes. Another study found that $N-3$ substituted compounds were nontoxic (Tang et al., 2015). Thus, there is a growing need to elucidate the correct metabolic profile of TZDs, which will in turn contribute to clarification of the safety profile of these drugs. The aim of this study was to investigate the properties of the metabolism of 5-(indol-3-ylmethylene)-3-(4-methylbenzyl)-thiazolidine-2,4-dione (GQ-11, Fig. 1B), including metabolic stability and metabolite formation, using human and rat microsomes, and to compare these findings with those of pioglitazone.

\section{Material and Methods}

Chemicals and Reagents. The compound 5-((1H-indol-3-yl)methylene)-3(4-methylbenzyl)thiazolidine-2,4-dione (GQ-11) was synthesized at the Laboratory of Design and Drug Synthesis of the Federal University of Pernambuco (Recife, Brazil). The thiazolidine derivative was synthesized by the nucleophilic Michael addition of substituted 3-benzyl-thiazolidine-dione on substituted cyanoacrylate, to obtain thethiazolidine-2,4-diones (GQs) (Mourao et al., 2005; da Costa Leite et al., 2007). Pioglitazone, Jacobsen catalyst, formic acid, hydrogen peroxide, $\mathrm{MgCl}_{2}$, glucose-6-phosphate, NADP, and glucose-6-phosphate dehydrogenase were purchased from Sigma-Aldrich (St Louis, MO). NADPH was purchased from Santa Cruz Biotechnology (Dallas, TX). Acetonitrile (highperformance liquid chtomatography grade) was purchased from J. T. Baker (Mexico City, Mexico). Human liver microsomes (HLMs, code HMMC-PM) and rat liver microsomes (RLMs, code RTMC-PL) were purchased from Thermo Scientific (Waltham, MA). Water was purified by a Millipore system (Merck Millipore, Burlington, MA).

Microsomal Incubation and Processing of Samples. HLMs or RLMs were thawed and diluted to appropriate protein concentrations $(0.5 \mathrm{mg} / \mathrm{ml})$ with $100 \mathrm{mM}$ potassium phosphate buffer $\left(\mathrm{pH} 7.4\right.$ at $\left.37^{\circ} \mathrm{C}\right)$. Incubations were started by the addition of NADPH $(1 \mathrm{mM})$ and compounds in two concentrations: $2 \mu \mathrm{M}$ for metabolic stability and $10 \mu \mathrm{M}$ for metabolite investigation. Final incubation volume was $1 \mathrm{ml}$. Incubations were carried out in triplicate in a 12-well plate. In the metabolic stability study, aliquots $(100 \mu \mathrm{l})$ were collected at time points 0,5 , 10, 20, 40, 60, and 90 minutes. For metabolite investigation, time point 0 was collected as the control sample, and the 60-minute time point was the test sample. Incubation was stopped by starting the sample processing.
For the investigation of metabolic stability, sample incubation media (100 $\mu 1)$ processing was started by the addition of ethyl acetate $(1 \mathrm{ml})$ and internal standard (10 $\mu \mathrm{l}$ ). The internal standard was rosiglitazone at $10 \mu \mathrm{g} / \mathrm{ml}$ for pioglitazone analysis and $100 \mu \mathrm{g} / \mathrm{ml}$ for GQ-11 analysis. Next, the mixture was vortex-mixed for 30 seconds and was then centrifuged for 5 minutes at $4000 \mathrm{~g}$, and the organic phase $(900 \mu \mathrm{l})$ was transferred to a new clean 1.5-ml microtube, which was evaporated to dryness using a MiVac concentrator (Genevac, Ipswich, Reino Unido) at $50^{\circ} \mathrm{C}$ for 40 minutes. The extract was resuspended with $50 \mu \mathrm{l}$ of mobile phase and 30 seconds of vortex-mixing. This mixture was transferred to a vial with an insert and placed in the sample manager until injection for the liquid chromatography (LC)-tandem mass spectrometry (MS/MS). For the investigation of metabolites, the previously described procedure, without internal standard, was used in addition to another protein precipitation procedure, resulting in two extracts for each sample. The protein precipitation consisted of adding $100 \mu \mathrm{l}$ of acetonitrile to $100 \mu \mathrm{l}$ of incubation media, followed by vortex-mixing and centrifugation at $15,000 \mathrm{~g}$ for 15 minutes. Next, the supernatant was separated and transferred to maximum recovery vials to be injected in the ultraperformance LC coupled to a hybrid quadrupole-time of flight mass spectrometer.

LC-Tandem MS Quantitation. Quantitation of pioglitazone and GQ-11 was performed on an LC Alliance 2695 (Waters, Milford, MA) coupled to a mass spectrometer triple-quadrupole Quattro Micro (Micromass, Wilmslow, UK) equipped with an electrospray ionization (ESI) interface. Data were acquired by using MassLynx 4.1 (Waters).

Chromatographic separation was performed using a Symmetry (Waters) C18 column $(50 \times 2.1 \mathrm{~mm}, 3.5 \mu \mathrm{m})$ protected by a $5 \times 2.1-\mathrm{mm}$ guard column of the same stationary phase, both at $35^{\circ} \mathrm{C}$. The flow rate was $0.3 \mathrm{ml} / \mathrm{min}$. Injection volume was $10 \mu \mathrm{l}$. For pioglitazone, separation was achieved using isocratic mobile phase consisting of acetonitrile: water (80:20); for GQ-11, the mobile phase was composed of acetonitrile and $2 \mathrm{mM}$ ammonium acetate (80:20).

MS parameters for the analysis were as follows. Positive ion mode was used for pioglitazone, and negative ion mode was used for GQ-11. ESI source and desolvation temperatures were 120 and $200^{\circ} \mathrm{C}$, respectively; capillary voltage was $4.5 \mathrm{kV}$ for pioglitazone and $3.0 \mathrm{kV}$ for GQ-11. Nitrogen was the desolvation gas $(800 \mathrm{l} / \mathrm{h})$, and argon was the collision gas. Analysis was performed working in multiple reaction monitoring mode. In the pioglitazone analysis, the transition $357>134$ was used for pioglitazone, $358>94$ for rosiglitazone. Cone voltage was $35 \mathrm{~V}$ for both, and collision was 23 and 40 (arbitrary units) for pioglitazone and rosiglitazone, respectively. In the GQ-11 analysis, the transitions were $347>172$ for GQ-11 and $356>313$ for rosiglitazone. Cone voltages were $33 \mathrm{~V}$ for GQ-11 and $25 \mathrm{~V}$ for rosiglitazone. Collision energy was 23 and 19 (arbitrary units) for GQ-11 and rosiglitazone, respectively.

Metabolic Stability Calculations. GQ-11 and pioglitazone concentrations were used to build the concentration versus time plot. Next, in vitro microsomal half-life, predicted intrinsic clearance $\left(\mathrm{CL}_{\mathrm{int}}\right)$, and predicted hepatic clearance $\left(\mathrm{CL}_{\mathrm{h}}\right)$ were calculated according to Obach (1999). For human microsomes, the assumptions were $45 \mathrm{mg}$ of microsomes per gram of liver, $20 \mathrm{~g}$ of liver per kilogram of body weight, and $21 \mathrm{ml} / \mathrm{min}$ per kilogram $(1470 \mathrm{ml} / \mathrm{min})$ as human hepatic blood flow (Obach, 1999); for rats, the assumptions were $45 \mathrm{mg}$ of microsomes per gram of liver, $45 \mathrm{~g}$ of liver per kilogram of body weight (Lu et al., 2006), and $55 \mathrm{ml} / \mathrm{min}$ per kilogram (220 ml/min) (Huang et al., 2015) as rat hepatic blood flow.

Biomimetic Oxidation Study Using the Jacobsen Catalyst. Reactions were carried out in a 2-ml microtube at room temperature for 24 hours and under mechanical stirring. Hydrogen peroxide was the oxygen donor, and Jacobsen catalyst was responsible for the catalysis. The molar ratio was 1:80:20 for catalyst:oxidant:drug. To achieve that proportion, the mixture was constituted by $6.0 \times 10^{-7} \mathrm{~mol}$ of catalyst, $4.8 \times 10^{-5} \mathrm{~mol}$ of oxygen donor, and $1.2 \times 10^{-5} \mathrm{~mol}$ of drug in the reaction medium. The reaction medium was acetonitrile $\left(\mathrm{CH}_{3} \mathrm{CN}\right)$. At the end of the reaction, agitation was stopped, and the reaction mixture was withdrawn. The products were analyzed by LC coupled to high- resolution MS.

LC-High-Resolution MS Analysis. Investigation of metabolites was performed using the Acquity H-Class UPLC system (Waters) coupled to a G2-S hybrid quadrupole-time of flight mass spectrometer (Waters) equipped with an ESI source operating in positive-ion mode. Data acquisition was carried out with MassLynx 4.1 (Waters).

The separation was performed on an Acquity HSS SB C18 column $(2.1 \times 100 \mathrm{~mm}$; $1.7 \mu \mathrm{m})$ protected by a HSS VanGuard guard column $(2.1 \times 5 \mathrm{~mm} ; 1.7 \mu \mathrm{m})$ at $35^{\circ} \mathrm{C}$. The gradient mobile phase solvents were water (A) and acetonitrile (B), both 
TABLE 1

In vitro metabolism parameters of pioglitazone and GQ-11, both at $2 \mu \mathrm{M}$, incubated with $0.5 \mathrm{mg} / \mathrm{ml} \mathrm{microsome} \mathrm{protein}$

\begin{tabular}{lccccc}
\hline Compound & Species & $k$ & $t_{1 / 2}$ & $\mathrm{CL}_{\text {int,predicted }}$ & $\mathrm{CL}_{\mathrm{h}, \text { predicted }}$ \\
\hline \multirow{4}{*}{ Pioglitazone } & & $\min ^{-1 a}$ & $\min$ & $\mathrm{ml} / \mathrm{kg} \mathrm{per} \mathrm{min}^{\mathrm{b}}$ & $\mathrm{ml}^{\mathrm{kg} \mathrm{per} \mathrm{min}} \mathrm{per}^{\mathrm{c}}$ \\
& Rat & $0.008 \pm 0.001$ & $93.2 \pm 13.7$ & $30.5 \pm 4.0$ & $19.6 \pm 1.7$ \\
GQ-11 & Human & $0.009 \pm 0.001$ & $78.7 \pm 8.2$ & $15.9 \pm 1.6$ & $9.0 \pm 0.5$ \\
& Rat & $0.0174 \pm 0.007$ & $44.7 \pm 18.3$ & $70.3 \pm 28.7$ & $30.0 \pm 5.6$ \\
& Human & $0.026 \pm 0.0001$ & $27.1 \pm 0.1$ & $46.1 \pm 0.3$ & $14.4 \pm 0.02$ \\
\hline
\end{tabular}

${ }^{a}$ Decay rate constant.

${ }^{b}$ Predicted intrinsic clearance.

Predicted hepatic clearance was obtained disregarding binding values.

containing formic acid $0.1 \%$ at a flow rate of $0.3 \mathrm{ml} / \mathrm{min}$. The initial mobilephase composition was maintained at $90 \%$ A for 1 minute, changed linearly to $10 \%$ (1-8 minutes) and held for 1 minute, and then returned to the initial condition at 10 minutes, and kept until 12 minutes (total running time) for the chromatograph column equilibrium. The injection volume was $5 \mu \mathrm{l}$.

The mass spectrometer ESI source capillary voltage was $4 \mathrm{kV}$. The temperature of the source was set at $150^{\circ} \mathrm{C}$, and the desolvation temperature was set at $350^{\circ} \mathrm{C}$. The cone voltage was $20 \mathrm{~V}$. Nitrogen was used as the cone and desolvation gas. The cone gas flow was 50 liters/h, and the desolvation gas flow was 550 liters/h. Leucine-enkephalin was used as the lock mass, generating a reference ion in positive mode at $\left(\mathrm{m} / \mathrm{z} 556.2771[\mathrm{M}+\mathrm{H}]^{+}\right)$, and introduced by a lock spray at $20 \mu \mathrm{l} / \mathrm{min}$ for accurate mass acquisition. For control and test sample analysis, data were collected using $\mathrm{MS}^{\mathrm{E}}$ in the scan range 50-600 Da. Function 1 with low energy was used to monitor precursor ions; function 2 with high-energy (ramp collision energy from 20 to $60 \mathrm{~V}$ ) was used to monitor product ions. After each pair of control and test samples, datadependent MS/MS experiments were performed for selected precursor ions using ramp collision energy from 10 to 40 (arbitrary units). Data acquisition was performed using MassLynx 4.1 software (Waters Corp).

Data Analysis. The $\mathrm{MS}^{\mathrm{E}}$ data were analyzed using Metabolynx XS, an extension of MassLynx software (Waters Corp.). The mass defect filter was enabled at $25 \mathrm{mDa}$, and the analysis time was 1-11 minutes. The mass window was $0.02 \mathrm{Da}$, the absolute area of the peak was 30 (p.a.u.), the spectrum was above the relative intensity of $2 \%$, and the maximum tolerance of mass error was set as $5 \mathrm{ppm}$. The prediction rules of elemental composition were defined as follows: atom numbers of carbon, hydrogen, oxygen, nitrogen, and sulfur were set to ranges of $0-100,0-120,0-60,0-10$, and 0-6, respectively. After processing, a new run of the test sample was performed using the metabolite peaks found as

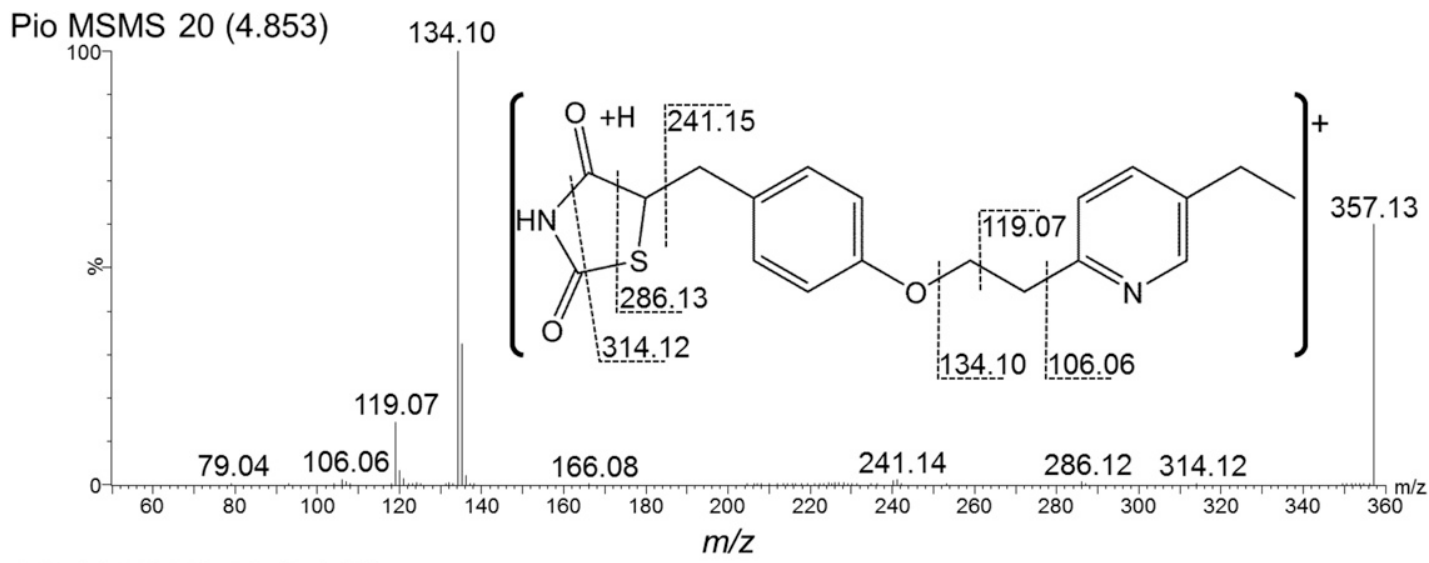

\section{GQ-11 MSMS 20 (6.110)}

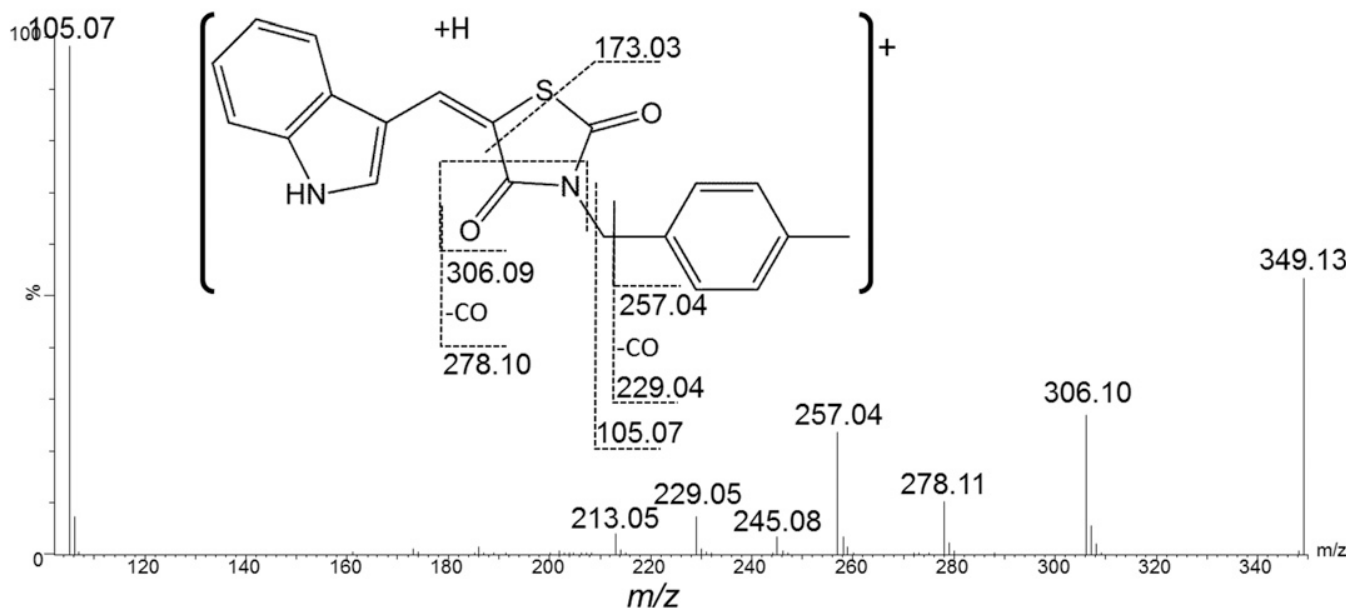

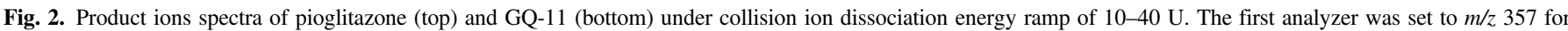
pioglitazone and $m / z 349$ for GQ-11. 
precursor ions in the MS/MS experiment to confirm the fragmentation pattern observed in the $\mathrm{MS}^{\mathrm{E}}$ acquisition mode.

\section{Results}

\section{Microsome Metabolic Stability}

Pioglitazone and GQ-11 were quantitated, and their concentrations were converted to remaining percentage and then plotted against time. The in vitro metabolism parameters can be seen in Table 1 . The free fraction in blood was not accounted for in scaling the clearance values to body weight.

\section{MS Analysis of Parent Drugs}

The analysis of parent compounds GQ-11 and pioglitazone was performed first to obtain their fragmentation pattern. The high-resolution MS analysis of GQ-11 and pioglitazone showed the $[\mathrm{M}+\mathrm{H}]^{+}$of both compounds with $\mathrm{m} / \mathrm{z} 349.1015$ and 357.1273 (1.1 and $1.7 \mathrm{ppm}$ ), respectively. The MS/MS analyses of pioglitazone and GQ-11 are shown in Fig. 2. Most of the product peaks were related to structure fragments. As can be observed, the base fragment peaks were 134 for pioglitazone and 105 for GQ-11. This is important as changes in the $\mathrm{m} / \mathrm{z}$ of specific parts of the molecule may be related to the expected metabolic reaction. In addition, other fragments may arise after metabolic structure modifications.

\section{Pioglitazone Metabolites}

Pioglitazone was incubated with human and rat microsomes to obtain its phase 1 metabolites and was compared with GQ-11 metabolite formation under the same conditions. Several metabolites of pioglitazone have been described in the literature (Shen et al., 2003; Uchiyama et al., 2010), and most were observed in the current study (Fig. 3; Table 2).

Well Established Pioglitazone Metabolites. Most of the observed metabolites were those reported in literature (Shen et al., 2003; Baughman et al., 2005; Uchiyama et al., 2010) and confirmed by exact mass, with error less than $5 \mathrm{ppm}$ (Table 2); $\mathrm{MS}^{2}$ product ions were in agreement with their structures.

A

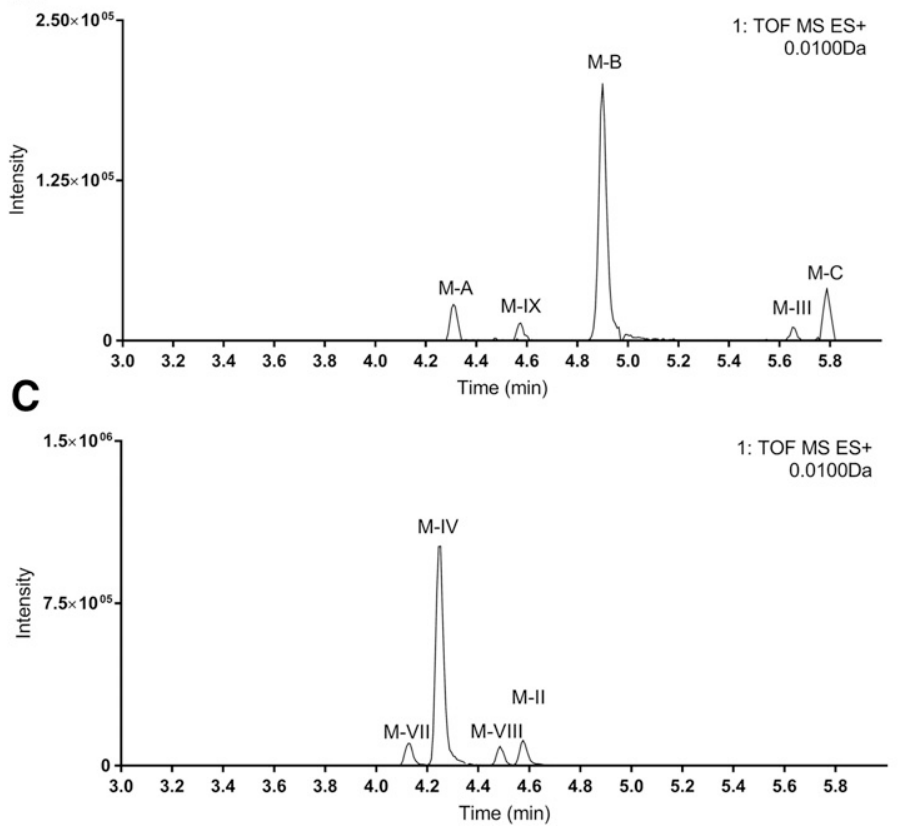

The metabolites formed by opening of the thiazolidinedione ring at 4.9 (M-B) and 5.79 minutes (M-C) were first described by Uchiyama et al. (2010) as S9P1 and M11, respectively. Uchiyama et al. (2010) suggested that the formation of S9P1 and M11 occurred with the $\mathrm{N}$-glucuronide metabolite (M7) as precursor since they observed the formation of S9P1 and M11 in an S9 metabolism experiment system containing S-adenosyl-L-methionine, and using M7 as the substrate; however, the conversion was not dependent on S-adenosyl-L-methionine or human liver S9. In our study, we have confirmed the occurrence of these metabolites but without the $N$-glucuronide metabolite M7, which suggests that these metabolites may also be formed by another pathway.

New Pioglitazone Metabolites. Two new pioglitazone metabolites were observed in the current study, a new hydroxylated metabolite at 5.9 minutes (M-D) and another metabolite resulting from hydration reaction at retention time 4.31 minutes (M-A). The former was observed only in the RLMs, whereas M-A was observed in both species. The base product ion of M-D was at $m / z 150$ (Fig. 4), indicating that an oxygen was added in the pyridine ring or in the carbon adjacent to the ether function. The fragmentation pattern suggests that the substitution site is aliphatic and not aromatic; however, it is not definitive proof, and further studies may confirm this observation. The metabolite formed by hydrolysis (M-A) of the thiazolidinedione ring may work as an intermediate between $\mathrm{M}-\mathrm{B}$ and $\mathrm{M}-\mathrm{C}$. The absence of the metabolite M-A (4.31 minutes) in previous studies may be explained by its proximity to the third isotope of the major hydroxylated metabolite (4.24 minutes). The protonated M-A $\left(\mathrm{C}_{19} \mathrm{H}_{23} \mathrm{~N}_{2} \mathrm{O}_{4} \mathrm{~S}^{+}\right)$has an $\mathrm{m} / z$ of 375.1379 . The protonated hydroxylated metabolite of 4.24 minutes $\left(\mathrm{C}_{19} \mathrm{H}_{21} \mathrm{~N}_{2} \mathrm{O}_{4} \mathrm{~S}^{+}\right)$was observed at the $\mathrm{m} / z$ of 373.1222 , but its third isotope peak (4.5\% intensity) appeared at $\mathrm{m} / \mathrm{z}$ of 375.1180 . This peak $(\mathrm{m} / \mathrm{z}, 375.1180)$ had an intensity as high as the major isotope of M-A. Therefore, to see it, it was necessary to close, as much as possible, the $\mathrm{m} / \mathrm{z}$ window to filter the interfering signal. The metabolite M-A has not been previously described; thus, its detailed spectrum is shown in Fig. 4. The fragmentation pattern is similar to that of the $N$-glucuronide metabolite described by Uchiyama et al. (2010). Our findings suggest an alternative pathway for formation of the openring metabolites $\mathrm{M}-\mathrm{B}$ and $\mathrm{M}-\mathrm{C}$ via hydrolysis of the imide in the

B
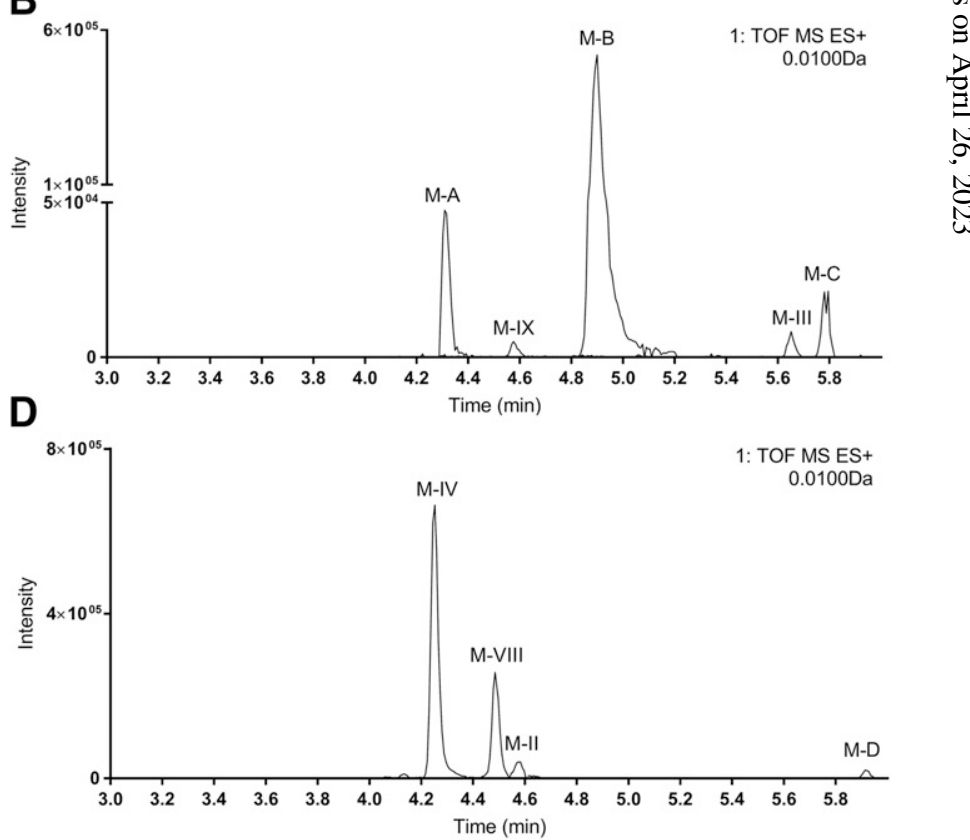

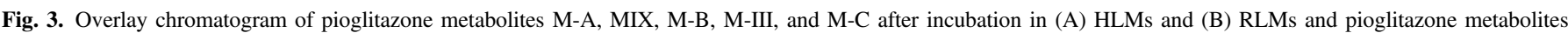
M-VII, M-IV, M-VIII, M-II, and M-D in (C) HLMs and (D) RLMs 
TABLE 2

Pioglitazone metabolites using ultraperformance LC coupled to hybrid quadrupole-time of flight after human and rat liver microsome incubation

Plus (+) sign indicates the presence of the metabolite in the respective microsome system.

\begin{tabular}{|c|c|c|c|c|c|c|}
\hline Metabolite $^{a}$ & $\mathrm{M}+\mathrm{H}$ & Error & Retention Time & Product Ions & RLMs & HLMs \\
\hline & $m / z$ & $m / z$ & $\min$ & $m / z$ & & \\
\hline M-II (M10) & 373.1221 & -0.2 & 4.58 & $133,150,210,238,239,284,355$ & + & + \\
\hline M-III (M13) & 371.1075 & 2.4 & 5.65 & 148 & + & + \\
\hline M-IV (M5) & 373.1231 & 2.5 & 4.24 & $132,150,355$ & + & + \\
\hline M-VII (M9) & 373.1225 & 0.9 & 4.13 & $119,150,240,256$ & & + \\
\hline M-VIII & 373.1222 & 0.1 & 4.49 & 268,313 & + & + \\
\hline M-IX (M12) & 355.1120 & 1.1 & 4.58 & $133,210,238,239,284$ & & + \\
\hline M-B (S9P1) & 332.1320 & 0 & 4.90 & $119,134,286$ & + & + \\
\hline M-C (M11) & 346.1464 & -3.7 & 5.79 & $119,134,254$ & + & + \\
\hline M-D & 373.1215 & -1.8 & 5.90 & $93,138,150,357$ & + & \\
\hline M-A & 375.1373 & -1.4 & 4.31 & $134,286,332$ & + & + \\
\hline
\end{tabular}

${ }^{a}$ Metabolites (M) labeled as previously described by Shen et al. (2003) (roman numerals) or with our label (capital letters); in parentheses is the label used by Uchiyama et al. (2010).

thiazolidinedione ring, leading to formation of an $S$-carbamothioate and a carboxylic acid.

\section{GQ-11 Metabolites}

The new thiazolidinedione was incubated with human and rat microsomes to obtain its phase 1 metabolites. The main objective was to determine whether opened-ring metabolites, similar to those reported for glitazones, can occur under the same conditions. All possible reactions previously related to TZDs were inputted before data processing. Further, metabolites predicted by the software Metabolizer v.15.12.14 (Chemaxon, Budapeste, Hungria) were added for data processing. Five metabolites were found (Table 3) as oxidized products of GQ-11 (Fig. 5).

The GQ-11 metabolite M1 was found only in the HLM incubation. M2 was higher in RLMs than in HLMs, whereas M3, M4, and M5 showed similar patterns. Three other peaks were observed as possible metabolites (retention times 5.35, 5.77, and 6.04 minutes), but they could not be confirmed, and they were found only in RLMs. Figure 6 shows the product ions spectra and the proposed structures of the five observed metabolites.

To find more evidence for the formation of the nonaromatic metabolites of GQ-11, a reaction using the Jacobsen catalyst- $\mathrm{H}_{2} \mathrm{O}_{2}$ oxidation system was used. This allowed retention time comparison and better observation of the $\mathrm{MS}^{2}$ products for the metabolites M1 and M2. Still, it was possible to select the in-source fragment of M2 at $m / z, 347$, which is formed by loss of water in the hydroxylation site. Figure 5 shows the chromatograms of the reaction products M1 (C) and M2 (D). The signals of reaction products in the $\mathrm{MS}^{2}$ spectra match the signals observed in the $\mathrm{MS}^{2}$ spectra of the microsome incubation sample (Supplemental Fig. 1).

The metabolite M1 has the characteristic fragment of the benzylic portion of the parent molecule $(\mathrm{m} / \mathrm{z} 105)$, indicating that the oxidation was not in that portion; however, neither of the other fragments was similar to those of the parent molecule. Still, the fragment $\mathrm{m} / z .132$ was

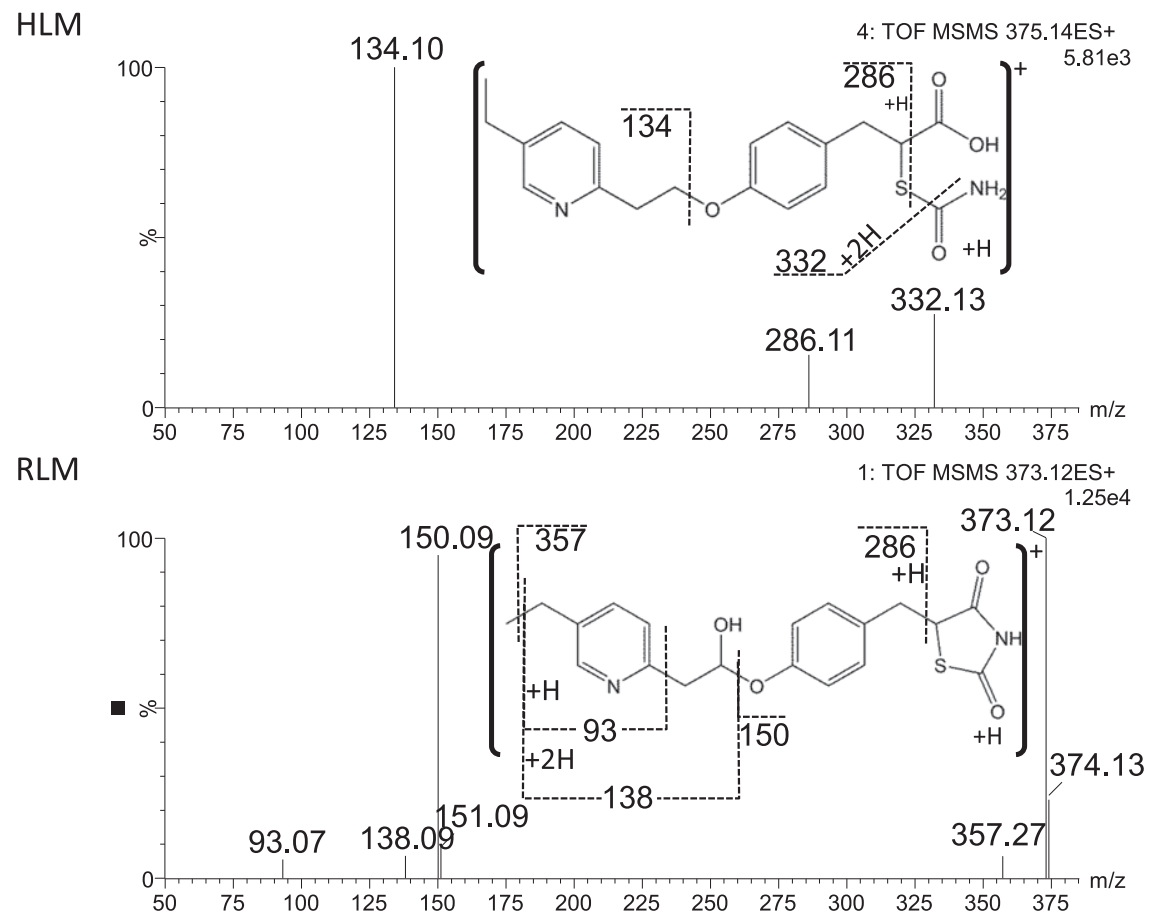

Fig. 4. Representative spectra and proposed fragmentation pattern of the metabolite M-A (top) $[\mathrm{M}+\mathrm{H}]^{+}$at $\mathrm{m} / \mathrm{z}$ $375\left(\mathrm{C}_{19} \mathrm{H}_{22} \mathrm{~N}_{2} \mathrm{O}_{4} \mathrm{~S}^{+}\right)$in HLMs and the metabolite M-D (bottom) $[\mathrm{M}+\mathrm{H}]^{+}$at $m / z 373\left(\mathrm{C}_{19} \mathrm{H}_{20} \mathrm{~N}_{2} \mathrm{O}_{4} \mathrm{~S}^{+}\right)$in RLMs. 
TABLE 3

GQ-11 metabolites using ultraperformance LC coupled to hybrid quadrupole-time of flight after human and rat liver microsome incubation

Plus (+) sign indicates presence of the metabolite.

\begin{tabular}{|c|c|c|c|c|c|c|}
\hline Metabolites & $\mathrm{M}+\mathrm{H}$ & Error & RetentionTime & Product Ions & RLMs & HLMs \\
\hline & $m / z$ & ppm & $\min$ & $\mathrm{m} / \mathrm{z}$ & & \\
\hline M1 & 365.0944 & -4.3 & 4.04 & 105,132 & & + \\
\hline M2 & 365.0948 & -3.2 & 4.20 & $121,173,319,347$ & + & + \\
\hline M3 & 365.0960 & 0.1 & 4.87 & $105,189,245,273,294,322$ & + & + \\
\hline M4 & 365.0964 & 1.2 & 4.97 & $105,189,245,273,294,322$ & + & + \\
\hline M5 & 365.0977 & 4.8 & 5.23 & $105,189,245,273,294,322$ & + & + \\
\hline
\end{tabular}

compatible with the presence of the oxygen in the indole portion of the molecule; however, the findings that support the occurrence of M3, M4, and M5 suggest that the oxygen is in the pyrrole ring of the indole portion (Fig. 6A). Also, the Jacobsen catalyst did not perform oxidation in the aromatic ring, and once M1 was formed in this reaction (Fig. 5C), this is evidence that M1 is not an aromatic hydroxylation.

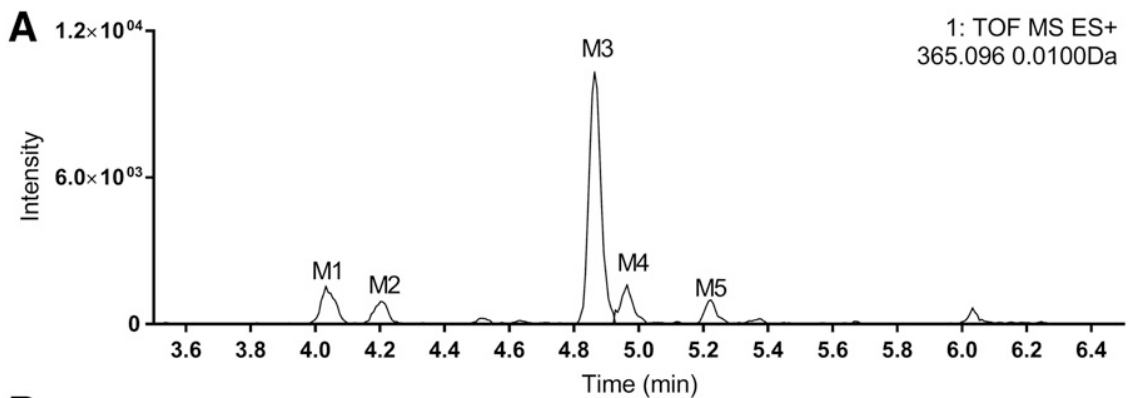

B

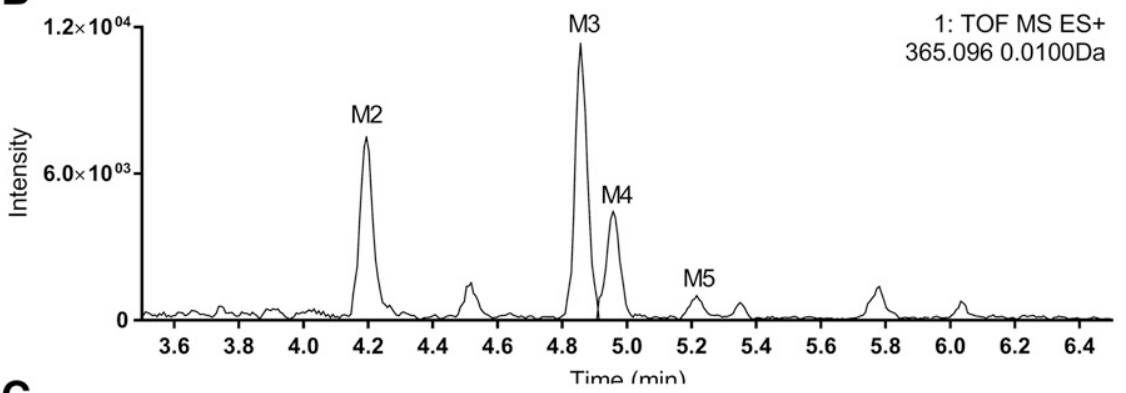

C
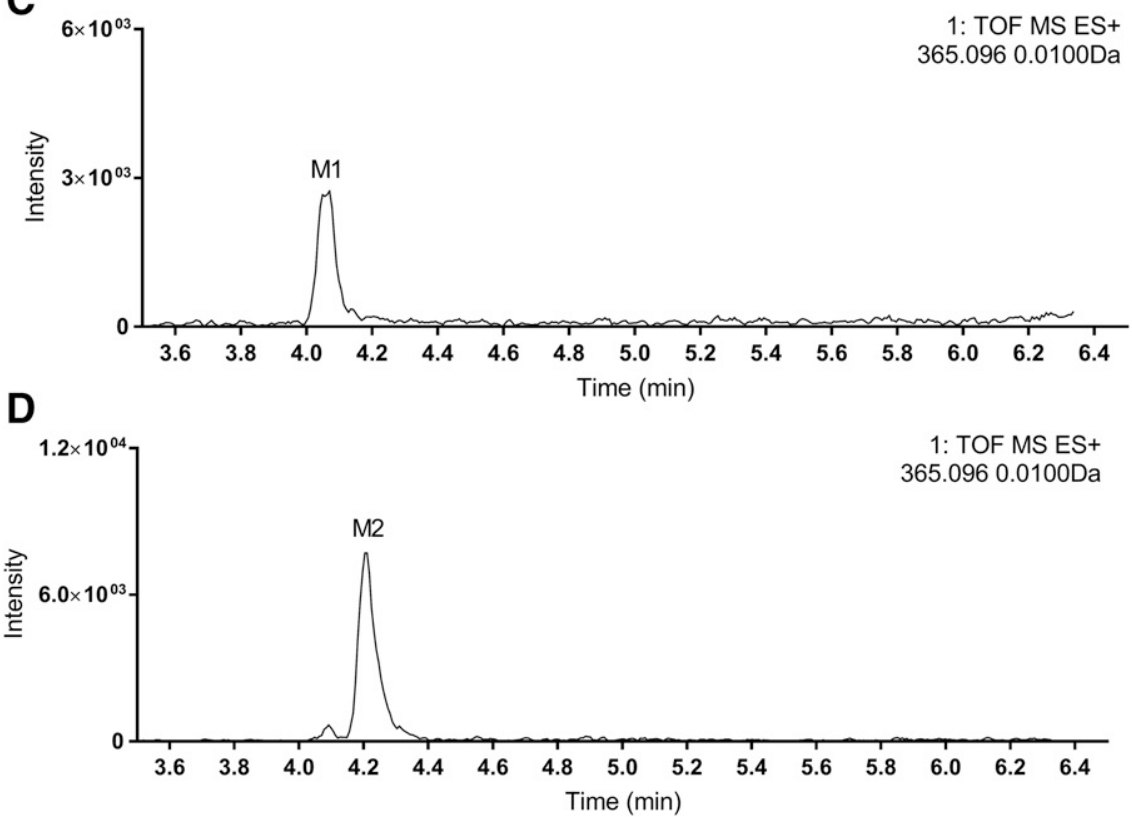

Fig. 5. Profile of the observed metabolites of GQ-11 after incubation in (A) HLMs, (B) RLMs, and after reaction using the Jacobsen catalyst- $\mathrm{H}_{2} \mathrm{O}_{2}$ system $(\mathrm{C}$ and $\mathrm{D})$. 


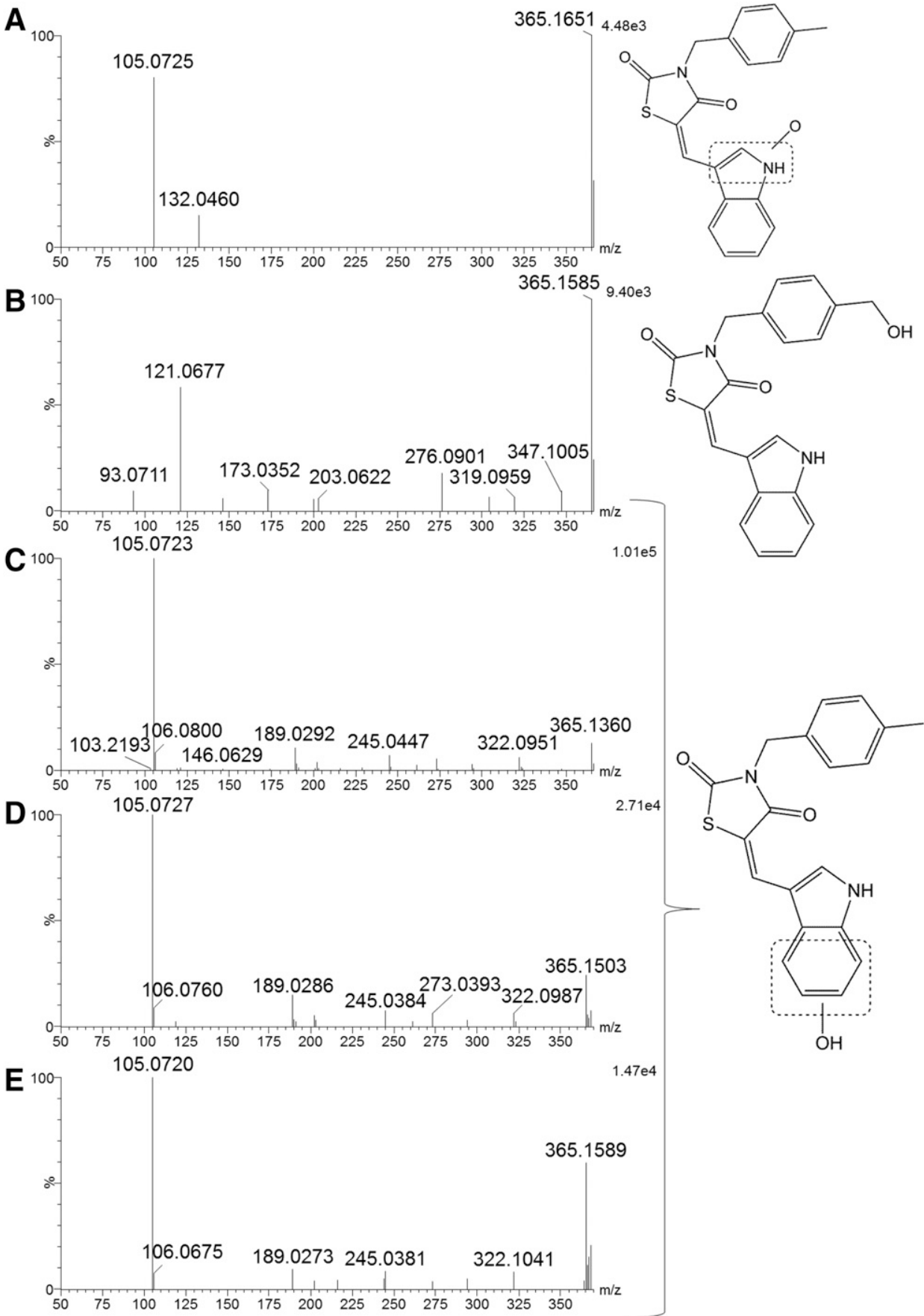

Fig. 6. Representative spectra and proposed structures of the oxidation metabolic products of GQ-11, M1 (A), M2 (B), M3 (C), M4 (D), and M5 (E).

The highest-intensity fragment of M2 (Fig. 6B) was in the $m / z, 121$, suggesting that the oxidation occurred in the benzylic portion of the molecule. The presence of the fragment $\mathrm{m} / \mathrm{z}, 347$ indicates the loss of $\mathrm{m} / \mathrm{z} 18$, probably a consequence of water loss, which is common for aliphatic alcohols. The reaction with Jacobsen catalyst was able to form M2 (Fig. 5D), and the $\mathrm{MS}^{2}$ fragments were in agreement with those of the microsome incubation system (Supplemental Fig. 1). Once $347\left[\mathrm{M}^{+}\right]$formed as an in-source fragment, it was used to help understand the structure of M2 by setting it as a parent ion to go through collision-induced dissociation. The main finding was the absence of $\mathrm{m} / \mathrm{z}, 121$ observed for the parent ion 365 and the rise of the $\mathrm{m} / \mathrm{z} 104$, corroborating the loss of neutral water in the parent ion. On the other hand, most of the other signals were similar between parent ions 347 and 365, also suggesting that the modification was in the benzylic portion.

The metabolites M3, M4, and M5 showed very similar fragmentation patterns (Fig. 6, C-E). The product ions matched the product ions of the parent molecule plus $16 \mathrm{Da}$, except the $\mathrm{m} / \mathrm{z} 105$, indicating that the oxidation was not in the benzylic part of the molecule. This similar pattern indicates that the metabolites are quite similar since the change in the position of the oxidation had no effect in the fragmentation mechanism of its peers. The only explanation for this is that the oxidation occurs in the aromatic portion of the indole ring, which is far from the fragmentation sites and would have the same effect in the molecule as a whole. The absence of M3, M4, and M5 in the Jacobsen catalyst reaction brings more evidence that they are aromatic hydroxylations. 


\section{Discussion}

TZDs such as pioglitazone are very useful for treatment of diabetes. An investigation of the metabolic stability of GQ-11 and pioglitazone was performed in rat and human liver microsomes. Pioglitazone had an intrinsic clearance around 3-fold greater than previously published (Xiao et al., 2015). The new candidate was less stable than pioglitazone in both microsome species. Considering these findings, it is important to highlight that there is a need for monitoring of metabolite formation. As expected, the predicted clearance for rats was higher than the predicted clearance for humans (Mahmood et al., 2003). The higher clearance of GQ-11 may lead to a half-life shorter than that of pioglitazone (8.3 hours) (Jaakkola et al., 2005). On the other hand, GQ-11 is more lipophilic ( $\log$ P 5.1) than pioglitazone ( $\log$ P 2.7) (Gimenez et al., 2010), which may help to extend its half-life in vivo by a change in volume of distribution.

The use of TZDs is limited by their toxicity, and some authors have related this toxicity to the formation of the thiazolidinedione ring opening metabolites in troglitazone (Kassahun et al., 2001), which is a common pathway for other TZDs (Shen et al., 2003; Baughman et al., 2005; Alvarez-Sánchez et al., 2006). These opened-ring metabolites have been previously reported (Uchiyama et al., 2010), and two of them (M-B and M-C) were observed in our work. In addition, a new hydration metabolic product (M-A) of pioglitazone was observed, which may be an intermediary in the formation of M-B and M-C. The new proposed hydration metabolite M-A eluted very close (4.31 minutes) to the retention time of the hydroxyl metabolite M-IV (4.25 minute), and was almost hidden because of this. Once the intensity of M-A was much smaller than the intensity of M-IV, it would be impossible to see it due to the presence of the $m / z, 375$ from the isotope pattern of the hydroxylated metabolite (M-IV m/z 373). Without the chromatographic separation, the $\mathrm{MS}^{2}$ fragments of $m / z, 375$ from M-A would be suppressed by the MS ${ }^{2}$ fragments of $m / z, 375$ from M-IV (monoisotopic $m / z, 373$ ). Although not reported before for pioglitazone, it would be predicted that hydrolysis in the imide function of the thiazolidinedione ring would occur, similar to what happens in the phthalimide ring of thalidomide with the formation of its $\mathrm{N}$-(o-carboxybenzoyl)glutamic acid imide metabolite (Lu et al., 2003). Another hydrolysis of imide was observed in aniracetam, which has a lactam ring, resulting in a cleaved product (Ogiso et al., 1998).

The other unpublished metabolite observed in the current study is proposed as the result of oxidation in the carbon adjacent to the ether group (Fig. 4), which is not related to opening in the thiazolidinedione ring; therefore, it may not be related to general toxicity. Indeed, some hydroxylation and oxidation products of pioglitazone, such as the metabolites M-III, M-IV, and M-II to a minor degree, share its hypoglycemic activity (Eckland and Danhof, 2000; Shen et al., 2003).

Based on the crucial role of TZDs in the treatment of diabetes and given the risk associated with the use of the available drugs of this class (Al-Salman et al., 2000; Maeda, 2001; Scheen, 2001, 2003; Marcy et al., 2004; Alemán-González-Duhart et al., 2016), the development of new safer TZDs is of current interest (Cesar et al., 2015; Rudnicki et al., 2016; Silva et al., 2016; Naim et al., 2017; Thangavel et al., 2017). Since glitazones do not cause liver damage in laboratory animals, comparing these with new TZDs to research the mechanism of toxicity can be misleading (Patel et al., 2012). Further, it has been shown that TZDs with the N3-carboxymethyl substituent are nontoxic (Tang et al., 2015), which may be related to their metabolic stability owing to avoidance of the ring opening since compounds with this feature are more stable than their peers. Considering the previously mentioned properties of pioglitazone metabolites, it is reasonable to assume that the absence of open-ring metabolites and the presence of hydroxylated metabolites for GQ-11 represent a promising profile for drug development.
Jacobsen catalyst is known to be able to react similarly to cytochrome $\mathrm{P} 450$ in the presence of an oxygen donor. Examples of this property can be found in literature, where it was reported to be able to react with drugs (Leod et al., 2007; Mac Leod et al., 2008) and natural products (De Santis Ferreira et al., 2012; Niehues et al., 2012; Rocha et al., 2014) to confirm or predict metabolism products. Therefore, the biomimetic system using Jacobsen catalyst and $\mathrm{H}_{2} \mathrm{O}_{2}$ was successfully applied to obtainment of the nonaromatic oxidation products of GQ-11 to bring more evidence for the proposed metabolite structures.

Only one metabolite signal was found in HLMs that was not found in RLMs; therefore, it will be necessary to look for the metabolite M1 in preclinical studies of GQ-11 since the metabolites identified only in human microsomes may arise safety concerns (FDA, 2008). Despite the uniqueness of M1, the similarity between the metabolite profiles in laboratory animals and humans may be enough to avoid the need to perform preclinical toxicologic studies for the preformed metabolites unless the formation of one of these metabolites surpasses $10 \%$ of total drug-related exposure (area under the curve). This is another important property for potentially successful new drug candidates (Baillie et al., 2002; FDA, 2008).

In summary, two new metabolites of pioglitazone were reported, one of them being a hydration product resulting from hydrolytic cleavage of the thiazolidinedione ring. The in vitro metabolic characteristics of the new thiazolidinedione GQ-11 were also described for the first time. Five new metabolites for GQ-11 were found, all products of oxidation reactions. The profile in human and rat liver microsomes was similar, with only one small-intensity metabolite being exclusively formed in human microsomes. Hydrolysis of the thiazolidinedione ring of GQ-11 was not observed, which suggests it is potentially less hazardous than the clinically available glitazones. This is probably a consequence of the substitution in the nitrogen at position 3 of the thiazolidinedione ring. Further metabolism studies with other N3-substituted thiazolidinediones may support this conclusion.

\section{Authorship Contributions}

Participated in research design: Campos, Pitta, Peccinini, Pontarolo. Conducted experiments: Campos, Cerqueira, Silva, Franchin, Galdino-Pitta. Contributed new reagents or analytic tools: Campos, Pitta, Peccinini, Pontarolo.

Performed data analysis: Campos.

Wrote or contributed to the writing of the manuscript: Campos, Cerqueira, Silva, Franchin, Galdino-Pitta, Pitta, Peccinini, Pontarolo.

\section{References}

Alemán-González-Duhart D, Tamay-Cach F, Álvarez-Almazán S, and Mendieta-Wejebe JE (2016) Current advances in the biochemical and physiological aspects of the treatment of type 2 diabetes mellitus with thiazolidinediones. PPAR Res 2016:7614270.

Al-Salman J, Arjomand H, Kemp DG, and Mittal M (2000) Hepatocellular injury in a patient receiving rosiglitazone: a case report. Ann Intern Med 132:121-124.

Alvarez-Sánchez R, Montavon F, Hartung T, and Pähler A (2006) Thiazolidinedione bioactivation: a comparison of the bioactivation potentials of troglitazone, rosiglitazone, and pioglitazone using stable isotope-labeled analogues and liquid chromatography tandem mass spectrometry. Chem Res Toxicol 19:1106-1116.

Amato AA, Rajagopalan S, Lin JZ, Carvalho BM, Figueira ACM, Lu J, Ayers SD, Mottin M, Silveira RL, Souza PCT, et al. (2012) GQ-16, a novel peroxisome proliferator-activated receptor $\gamma$ $(\operatorname{PPAR} \gamma)$ ligand, promotes insulin sensitization without weight gain. J Biol Chem 287: 28169-28179.

Araújo TG, Carvalho BM, Martins da Fonseca CS, de Lima Md CA, Galdino SL, da Rocha Pitta I and de Menezes Lima VL (2011) Metabolic effects of benzylidene thiazolidinedione derivatives in high-fat fed mice. Med Chem Res 21:2408-2414.

Baillie TA (2008) Metabolism and toxicity of drugs. Two decades of progress in industrial drug metabolism. Chem Res Toxicol 21:129-137.

Baillie TA, Cayen MN, Fouda H, Gerson RJ, Green JD, Grossman SJ, Klunk LJ, LeBlanc B, Perkins DG, and Shipley LA (2002) Drug metabolites in safety testing. Toxicol Appl Pharmacol 182:188-196.

Barros CD, Amato AA, de Oliveira TB, Iannini KB, Silva AL, Silva TG, Leite ES, Hernandes MZ, Alves de Lima Mdo C, Galdino SL, et al. (2010) Synthesis and anti-inflammatory activity of new arylidene-thiazolidine-2,4-diones as PPARgamma ligands. Bioorg Med Chem 18:3805-3811. 
Baughman TM, Graham RA, Wells-Knecht K, Silver IS, Tyler LO, Wells-Knecht M, and Zhao Z (2005) Metabolic activation of pioglitazone identified from rat and human liver microsomes and freshly isolated hepatocytes. Drug Metab Dispos 33:733-738.

Beuck S, Schänzer W, and Thevis M (2011) Investigation of the in vitro metabolism of the emerging drug candidate S107 for doping-preventive purposes. J Mass Spectrom 46:112-130.

Brandon EFA, Raap CD, Meijerman I, Beijnen JH, and Schellens JHM (2003) An update on in vitro test methods in human hepatic drug biotransformation research: pros and cons. Toxicol Appl Pharmacol 189:233-246.

César FA, Rudnicki M, de Las Heras B, Boscá L, Lima MC, Pitta IR, and Abdalla DS (2015) New indole-thiazolidine attenuates atherosclerosis in $\operatorname{LDLr}(-/-)$ mice. Vascul Pharmacol 71 174-180.

Chase MP and Yarze JC (2002) Pioglitazone-associated fulminant hepatic failure. Am J Gastroenterol 97:502-503

da Costa Leite LFC, Veras Mourão RH, Alves de Lima MC, Galdino SL, Hernandes MZ, de Assis Rocha Neves F, Vidal S, Barbe J, and da Rocha Pitta I (2007) Synthesis, biological evaluation and molecular modeling studies of arylidene-thiazolidinediones with potential hypoglycemic and hypolipidemic activities. Eur J Med Chem 42:1263-1271.

De Santis Ferreira L, Callejon DR, Engemann A, Cramer B, Humpf H-U, de Barros VP, Assis Md, da Silva DB, de Albuquerque S, Okano LT, et al. (2012) In vitro metabolism of grandisin, a lignan with anti-chagasic activity. Planta Med 78:1939-1941.

Eckland D and Danhof M (2000) Clinical pharmacokinetics of pioglitazone. Exp Clin Endocrino Diabetes 108:234-242.

Food and Drug Administration (FDA) (2008) Guidance for Industry Safety Testing of Drug Metabolites, pp 11, U.S. Department of Health and Human Services, Food and Drug Administration, Center for Drug Evaluation and Research, Rockville, MD.

Forman LM, Simmons DA, and Diamond RH (2000) Hepatic failure in a patient taking rosiglitazone. Ann Intern Med 132:118-121.

Giménez BG, Santos MS, Ferrarini M, and Fernandes JP (2010) Evaluation of blockbuster drugs under the rule-of-five. Pharmazie 65:148-152.

Hauner H (2002) The mode of action of thiazolidinediones. Diabetes Metab Res Rev 18 (Suppl 2): $\mathrm{S} 10-\mathrm{S} 15$.

Imran M, Ilyas B, Deepanjali, and Khan SA (2007) Recent thiazolidinediones as antidiabetics. J Sci Ind Res (India) 66:99-109.

Jaakkola T, Backman JT, Neuvonen M, and Neuvonen PJ (2005) Effects of gemfibrozil, itraconazole, and their combination on the pharmacokinetics of pioglitazone. Clin Pharmacol Ther 77: 404-414.

Kassahun K, Pearson PG, Tang W, McIntosh I, Leung K, Elmore C, Dean D, Wang R, Doss G, and Baillie TA (2001) Studies on the metabolism of troglitazone to reactive intermediates in vitro and in vivo. Evidence for novel biotransformation pathways involving quinone methide formation and thiazolidinedione ring scission. Chem Res Toxicol 14:62-70.

Kostiainen R, Kotiaho T, Kuuranne T, and Auriola S (2003) Liquid chromatography/atmospheric pressure ionization-mass spectrometry in drug metabolism studies, $J$ Mass Spectrom 38 357-372.

Leclercq L, Cuyckens F, Mannens GSJ, de Vries R, Timmerman P, and Evans DC (2009) Which human metabolites have we MIST? Retrospective analysis, practical aspects, and perspectives for metabolite identification and quantification in pharmaceutical development. Chem Res Toxicol 22:280-293.

Leod TCOM, Barros VP, Faria AL, Schiavon MA, Yoshida IVP, Queiroz MEC, and Assis MD (2007) Jacobsen catalyst as a P450 biomimetic model for the oxidation of an antiepileptic drug. J Mol Catal A Chem 273:259-264.

Huang Z, Li H, Zhang Q, Tan X, Lu F, Liu H, and Li S (2015) Characterization of preclinical in vitro and in vivo pharmacokinetics properties for KBP-7018, a new tyrosine kinase inhibitor candidate for treatment of idiopathic pulmonary fibrosis. Drug Des Devel Ther 9 4319-4328

Lu C, Li P, Gallegos R, Uttamsingh V, Xia CQ, Miwa GT, Balani SK, and Gan L-S (2006) Comparison of intrinsic clearance in liver microsomes and hepatocytes from rats and humans: evaluation of free fraction and uptake in hepatocytes. Drug Metab Dispos 34 $1600-1605$

Lu J, Palmer BD, Kestell P, Browett P, Baguley BC, Muller G, and Ching LM (2003) Thalidomide metabolites in mice and patients with multiple myeloma. Clin Cancer Res 9:1680-1688.

Mac Leod TCO, Faria AL, Barros VP, Queiroz MEC, and Assis MD (2008) Primidone oxidation catalyzed by metalloporphyrins and Jacobsen catalyst. J Mol Catal A Chem 296:54-60.

Maeda K (2001) Hepatocellular injury in a patient receiving pioglitazone. Ann Intern Med 135:306

Mahmood I, Green MD, and Fisher JE (2003) Selection of the first-time dose in humans: comparison of different approaches based on interspecies scaling of clearance. J Clin Pharmacol 43 692-697.
Marcy TR, Britton ML, and Blevins SM (2004) Second-generation thiazolidinediones and hepatotoxicity. Ann Pharmacother 38:1419-1423.

Masimirembwa CM, Bredberg U, and Andersson TB (2003) Metabolic stability for drug discovery and development: pharmacokinetic and biochemical challenges. Clin Pharmacokinet 42:515-528.

Mourão RH, Silva TG, Soares ALM, Vieira ES, Santos JN, Lima MCA, Lima VLM, Galdino SL, Barbe J, and Pitta IR (2005) Synthesis and biological activity of novel acridinylidene and benzylidene thiazolidinediones. Eur J Med Chem 40:1129-1133.

Naim MJ, Alam MJ, Ahmad S, Nawaz F, Shrivastava N, Sahu M, and Alam O (2017) Therapeutic journey of 2,4-thiazolidinediones as a versatile scaffold: an insight into structure activity relationship. Eur J Med Chem 129:218-250.

Niehues M, Barros VP, Emery FdaS, Dias-Baruffi M, Assis Md, and Lopes NP (2012) Biomimetic in vitro oxidation of lapachol: a model to predict and analyse the in vivo phase I metabolism of bioactive compounds. Eur J Med Chem 54:804-812.

Obach RS (1999) Prediction of human clearance of twenty-nine drugs from hepatic microsoma intrinsic clearance data: an examination of in vitro half-life approach and nonspecific binding to microsomes. Drug Metab Dispos 27:1350-1359.

Ogiso T, Iwaki M, Tanino T, Ikeda K, Paku T, Horibe Y, and Suzuki H (1998) Pharmacokinetics of aniracetam and its metabolites in rats. J Pharm Sci 87:594-598.

Patel NN, Crincoli CM, Frederick DM, Tchao R, and Harvison PJ (2012) Effect of structural modifications on 3-(3,5-dichlorophenyl)-2,4-thiazolidinedione-induced hepatotoxicity in Fischer 344 rats. $J$ Appl Toxicol 32:108-117.

Rocha BA, de Oliveira ARM, Pazin M, Dorta DJ, Rodrigues APN, Berretta AA, Peti APF, de Moraes LAB, Lopes NP, Pospísil S, et al. (2014) Jacobsen catalyst as a cytochrome P450 biomimetic model for the metabolism of monensin A. BioMed Res Int 2014:152102.

Rudnicki M, Tripodi GL, Ferrer R, Boscá L, Pitta MGR, Pitta IR, and Abdalla DSP (2016) New thiazolidinediones affect endothelial cell activation and angiogenesis. Eur J Pharmacol 782:98-106

Saltiel AR and Olefsky JM (1996) Thiazolidinediones in the treatment of insulin resistance and type II diabetes. Diabetes $\mathbf{4 5}$ :1661-1669.

Santin JR, Daufenback Machado I, Rodrigues SF, Teixeira S, Muscará MN, Lins Galdino S, da Rocha Pitta I, and Farsky SH (2013a) Role of an indole-thiazolidine molecule PPAR panagonist and COX inhibitor on inflammation and microcirculatory damage in acute gastric lesions. PLoS One 8:e76894.

Santin JR, Uchôa FD, Lima MdoC, Rabello MM, Machado ID, Hernandes MZ, Amato AA, Milton FA, Webb P, Neves FdeA, et al. (2013b) Chemical synthesis, docking studies and biological effects of a pan peroxisome proliferator-activated receptor agonist and cyclooxygenase inhibitor. Eur J Pharm Sci 48:689-697.

Scheen AJ (2001) Hepatotoxicity with thiazolidinediones: is it a class effect? Drug Saf 24:873-888.

Scheen AJ (2003) Reduced potential for hepatotoxicity with newer thiazolidinediones? Inpharma Weekly 1379: 19-20.

Shen Z, Reed JR, Creighton M, Liu DQ, Tang YS, Hora DF, Feeney W, Szewczyk J, Bakhtiar R, Franklin RB, et al. (2003) Identification of novel metabolites of pioglitazone in rat and dog. Xenobiotica 33:499-509.

Silva JC, César FA, de Oliveira EM, Turato WM, Tripodi GL, Castilho G, Machado-Lima A, de Las Heras B, Boscá L, Rabello MM, et al. (2016) New PPAR $\gamma$ partial agonist improves obesityinduced metabolic alterations and atherosclerosis in LDLr(-/-) mice. Pharmacol Res 104:49-60.

Stumvoll M (2003) Thiazolidinediones - some recent developments. Expert Opin Investig Drugs 12:1179-1187.

Tang SQ, Lee YYI, Packiaraj DS, Ho HK, and Chai CLL (2015) Systematic evaluation of the metabolism and toxicity of thiazolidinone and imidazolidinone heterocycles. Chem Res Toxicol 28:2019-2033.

Thangavel N, Al Bratty M, Akhtar Javed S, Ahsan W, and Alhazmi HA (2017) Targeting peroxisome proliferator-activated receptors using thiazolidinediones: strategy for design of novel antidiabetic drugs. Int J Med Chem 2017:1069718.

Uchiyama M, Fischer T, Mueller J, Oguchi M, Yamamura N, Koda H, Iwabuchi H, and Izumi T (2010) Identification of novel metabolic pathways of pioglitazone in hepatocytes: $\mathrm{N}$-glucuronidation of thiazolidinedione ring and sequential ring-opening pathway. Drug Metab Dispos 38:946-956.

Xiao Q, Tang L, Xu R, Qian W, and Yang J (2015) Physiologically based pharmacokinetics model predicts the lack of inhibition by repaglinide on the metabolism of pioglitazone. Biopharm Drug Dispos 36:603-612.

Address correspondence to: Roberto Pontarolo, Department of Pharmacy, Universidade Federal do Paraná, 632 Lothário Meissner Avenue, 80210-170, Curitiba, Paraná, Brazil. E-mail: pontarolo@ufpr.br 